\title{
Formation of Multicultural Competence of Future Tourist Support Specialists (Guides) as a Pedagogical Problem
}

\author{
Oleksandr V. Kovalenko \\ Sumy State Pedagogical University Named After A.S. Makarenko \\ Larysa I. Yepyk \\ Sumy State Pedagogical University Named After A.S. Makarenko \\ Larysa V. Korzh-Usenko \\ Sumy State Pedagogical University Named After A.S. Makarenko \\ Oksana I. Skorobahatska \\ Sumy State Pedagogical University Named After A.S. Makarenko \\ Olga S. Oliinyk \\ Kamianets-Podilskyi Ivan Ohiienko National University
}

\begin{abstract}
The purpose of the paper is to study multicultural competency training future tourist support specialists (guides) in the higher educational institutions. Multicultural competency training is defined as ways of teaching and educating aimed at developing multicultural competency. It may include: creating awareness of one's cultural worldview, stereotypes and biases; deepening knowledge of different cultural practices and views; understanding and understanding that cultural and linguistic differences can affect communication; understanding why and how communication and behaviour should be adapted in different cross-cultural situations; how to work effectively and respectfully with people of different cultural and linguistic backgrounds; highlighting the benefits of diversity in the workplace or in society more broadly; creating plans and strategies for developing organisational multicultural competency, such as multicultural access and equity or hiring policy plans. At the university organisational level, developing multicultural competency requires clear and practical policies and procedures, as well as a commitment to implementation and evaluation to create a workplace that evaluates and matches cultural and linguistic diversity.
\end{abstract}

Keywords: tourism, guide training, professional training of specialists, multicultural, higher education

\section{INTRODUCTION}

Multicultural competency - the ability to understand, communicate and interact with people of other cultures effectively. Multicultural competency has organisational and individual aspects. At the individual 
level, the development of multicultural competency requires the recognition of one's cultural assumptions, values, and beliefs (Priulla et al., 2021). This involves understanding that culture shapes the worldview and that people see the world differently. Multicultural competency involves understanding that people have different needs. Improving multicultural competency provides better and more effective communication with people from different cultural and linguistic backgrounds (Chimienti et al., 2021). At the university organisational level, multicultural competency involves developing systems, policies, and processes that ensure cultural diversity in all aspects of the work. Higher educational organisations develop a successful diverse workforce with a high level of multicultural competency, using cultural differences as a force for more effective decision-making, innovation, and adaptation. They also understand the needs and preferences of a wide range of consumers and provide relevant, affordable, and comprehensive products and services. Culture is a dynamic, complex and constantly changing structure, there is no point at which cultural competence is achieved. Developing individual or organisational multicultural competency is an open process of improving attributes, skills, degrees of knowledge, policies, and strategies that together improve the ability to communicate between cultures.

Multicultural competency develops across a wide range, rather than by mastering a set of skills or practices. Developing multicultural competency requires a commitment to continuous learning, reflection, as well as diverse and inclusive practices (Santoso et al., 2021). Multiculturalists may find it useful to consider the fate of past efforts to improve the effectiveness of mental health services based on sociological data. After World War II, mental health professionals believed that society could be divided into classes. It was believed that the poor were both morally and functionally different from the more prosperous. Evidence was presented that the differential distribution of neurotic and psychotic patients by class was significant beyond the probability level, with the former being more common among the wealthier and the latter among the poorer. These results were seen as confirmation of the socio-Darwinian belief that people with lower socioeconomic status were abnormal, spoiled, unable to delay gratification, and so consciously aware that they suffered from frustrated aspirations for upper-class mobility. They were also considered related to their relatives and therefore had no individual responsibility, no rationality, and suffered from a relatively limited range of perceptions of the world around them (i.e., the middle-class world) (Dahinden and Anderson, 2021).

Guides have certain responsibilities as providers of the travel experience, sometimes competing with each other to meet the needs of visitors, employers, and host communities. Visitors expect their safety and health to come first, but at the same time expect a pleasant and rewarding travel experience. Some have special needs and expectations related to their cultural background, their physical and intellectual abilities, and their passions and interests in specific subjects. Employers expect the guide to provide high-quality service to visitors to meet these expectations, as well as manage the group, route, and other logistical aspects of the experience to maximise not only visitor satisfaction but also profit. Additional legal requirements may be provided for certain destinations, communities, and locations, as well as expectations that guides will give tours responsibly and ethically (Alam et al., 2020). In particular, ecotours, guides and nature monuments that guide visitors to protected areas are responsible for providing a high-quality interpretation of natural resources and heritage resources (Kovalenko, 2020). In addition to the stakeholders already mentioned, they may have obligations to conservation managers through licensing and accreditation systems.

The purpose of the paper is to study multicultural competency training future tourist support specialists (guides) as a pedagogical problem.

\section{MATERIALS AND METHODS}

During the study, the scientific literature was analysed. Based on the premise of determining the degree of literature, it is necessary to explain how to determine the authenticity of literature first. Methods for identifying literature include two methods: external audit and internal audit, respectively. External audit consists in identifying the reliability of literary sources. It is necessary to determine the authenticity of a version by comparing the style of presentation of the source with modern publications; on the other hand, 
it is also possible to check whether the contents of the book describe facts that occur after the book has been written since then. It is also worth identifying the authenticity. There are usually three methods used. The first is to analyse other works of an author to find out the author's language styles, and then check whether the language style corresponds to the available literature. The second is to analyse whether the style of literature is really sustained. Third, whether the idea of literature is consistent with the logical point of view. Internal audit refers to identifying the authenticity of content.

The main methods include: first, mutual word proof. If there is a discrepancy between the described events, it should be checked. Secondly, you can use a real object for literature in words. It is necessary to check whether the text description corresponds to the physical evidence. The third is to compare the content of documents with historical data to see if it corresponds to the current political and cultural background. The fourth is to study life, points of view, and main thoughts of the author, a specific environment in the formation of literature to determine the objectivity and trends of the author. Internal and external audits compare to determine the authenticity of content and to collect data on the quality of literature. To determine the authenticity of documents, you can evaluate the collected materials. For content processing. Thus, educational literature can be divided into zero grade, first grade, second grade, and third grade. Zero grade literature is commonly referred to as first-hand literature, it is the first description of events or experiences and is written by people who experience that event and represent the most primitive original documents that have not been published or modified, such as a letter, draft, draft discussion or original entry. Firstclass literature usually refers to works, recognised incidents, research results, new knowledge and new technologies, monographs, articles, investigation reports, and other literature.

This also can be secondary literature, which includes reports or materials describing evidence processed once by actual witnesses and participants of events. It includes books, newspapers, periodicals, research, reports, government publications, and so on. Second-grade literature is also known as document search. This refers to literature that processes and sorts books and articles to summarise the main points and put them in a system using some methods, such as bibliographies, titles, abstracts, indexes, and other publications. Such documentation will be distributed in the systematic literature and records. Third-grade literature is also known as reference literature. This systematically sorts the first-grade literature and compiles a resume based on the second-grade literature. Such literature differs from the original one, as well as from secondary literature. It is subjective in nature and reflects authors' opinions about scientific literature and is a complex result of many studies, literature such as dynamic synthesis of educational research, educational thematic comments, educational progress.

\section{RESULTS}

People with a high level of multicultural competency value diversity and can perform well in different cultural contexts. A higher educational institution can assess the current level of multicultural competency by analysing existing policies, staffing, and service delivery. The following can help identify gaps and risks and provide ways to improve (Dahinden et al., 2020). Organisations that value cultural diversity and cultural competence have updated, measurable and visible policies that promote cultural diversity of the workforce and provide inclusive services actively implement, monitor and evaluate diversity and inclusion policies to ensure organisational leaders' responsibility for diversity and results related to inclusion designate senior champions of cultural and linguistic diversity to explain and support policies that promote diversity, inclusion and cultural change (Ahmed et al. , 2020). Policies related to cultural and linguistic diversity can be included in many documents or combined into a single plan, such as multicultural access and equity plan. Although the format may vary from one organisation to another, good cultural and linguistic diversity policies should include a common goal or objectives and be supported by actions, initiatives, or results. They should also set a schedule and appoint a responsible department or senior employee.

The policy should include a vision or statement of cultural and linguistic diversity. This can be a short statement describing why the organisation decided to develop a plan and what they plan to achieve (Poglia Mileti, 2019). Higher educational institutions should formulate and address issues of cultural and linguistic diversity in the field of staffing and recruitment. They must outline how they will invite, retain and promote 
tourism workers. Hiring policies should also determine how multicultural skills will develop and be included in job selection criteria and performance reviews. If organisations need bilingual staff and translators, the recruitment policy may also require including these skills in job selection criteria, as well as details on how these additional skills will be recognised and rewarded. Strong cultural diversity policies should also be used to describe how services are designed, developed, and provided to respond to the diverse cultural and linguistic needs of the community. Organisations are comprehensive, accessible, and deliver fair results with a high level of multicultural competency clearly state how the services they provide. They can describe in detail how community engagement, surveys, feedback, or research will be used to incorporate the perspectives of tourism consumers into product and service development and reduce barriers to access (Spini et al., 2019).

Strong cultural diversity policies clearly define how and when monitoring and evaluation will take place. This includes who is responsible and how the results will be used to improve services. Strong cultural diversity policies include other supportive initiatives that promote cultural and linguistic diversity and inclusion. This may include special events, mentoring programmes, networks, scholarships, or internal communications that promote a culture of diversity and inclusion. It is important to note that strong cultural diversity policies are developed in conjunction with investments from all levels of the workforce, including senior managers. The senior managers must demonstrate a commitment to cultural and linguistic diversity through action and accountability. Culture is transmitted through enculturation, i.e. environmental influences that promote unconscious intrusion and conscious learning. Families play a key role as arbiters of the dominant culture and creators of their microcultures. Parenting is the last form of socialisation through which children learn to function in society. But parents vary in their ability and desire to pass on cultural beliefs to their children, and children are not passive recipients of parental values and practices. This explains the fact that the culture with which young people leave their families is rarely a copy of parental beliefs, which creates a variety of characters at each family reunion.

Immigration also affects the worldview, challenging the ethnic identity that the aliens came from. Ethnicity refers to "social groups that differ from other groups by sharing a common historical path, norms of behaviour, and the group's own identity" (Anderson, 2019). Immigrants can assimilate, move away from their ethnic heritage and immerse themselves in the general political channel, integrate these two sets of views, separate from the mainstream and accept only the beliefs of the heritage, or marginalise, do not accept or integrate any of the beliefs. Various factors influence acculturation, including the host culture's susceptibility to immigrants, the degree to which immigrant characteristics differ, and the extent to which native culture members will accept those who assimilate. Organisations that value cultural diversity and cultural competence reflect cultural and linguistic diversity at all levels of the organisation, including in senior management; collect and monitor data on the cultural and linguistic diversity of the workforce; monitor the preservation and development of diverse cultural and linguistic staff; have hiring strategies that ensure equal access to employment opportunities, address issues of racism, discrimination and unconscious prejudice and ensure the diversity of cultural and language teams; regularly review hiring strategies; provide opportunities for professional development, mentoring and role models for staff that is recognised culturally and ensure that multicultural and linguistic competence skills are a valuable component of professional development or performance. Multicultural competency training in higher educational institutions is a method of education aimed at creating multicultural competency. Training consists of the following components:

- understanding your cultural worldview, a set of stereotypes and biases;

- study of various cultural practices;

- awareness of cultural and linguistic differences that may affect communication;

- understanding how to adapt communication and behaviour in different cross-cultural situations;

- ensuring effective work with people of different cultural and linguistic backgrounds;

- highlighting the broad benefits of diversity in society;

- create tactics and strategies to ensure the development of multicultural competency. 
Multicultural competency training programmes in higher educational institutions can be advertised as inter-cultural awareness training; inter-cultural awareness training; multicultural sensitivity training; cultural diversity training; diversity and inclusion training; unconscious bias training; cross-cultural communication; cultural intelligence training; cultural ability training. The content depends on the programmes, depending on the needs of the organisation and the organisation that conducts the training. The content for teaching cultural competencies, which focuses on communication between cultures, is usually based on two academic foundations: "Theory of cultural dimensions" and "Seven dimensions of culture". Each of these theories uses dimensions, such as the importance of individualism versus communitarianism, or the impact of avoiding uncertainty (the tendency to avoid a situation that can cause discomfort) to analyse, classify, and understand a particular culture. At the same time, these theories help to structure communications so that the intended message is correctly understood by interlocutors from different cultures.

\section{DISCUSSION}

There is no standard model for teaching cultural competencies, and different programs are suitable for different organisations based on existing levels of multicultural competency, labour needs, and overall operations. Some high-quality programmes and instructors offer different approaches, content, delivery format, and duration. Although good cultural competence training programmes may look different, they have a number of things in common. Good multicultural competency training is based on evidence, research, and experience. Not all curricula include the same academic theory, but good programmes include content that has been developed using evidence and a consistent theoretical framework. They are based on relevant and up-to-date data and research from a wide range of reliable sources. Good multicultural competency curricula clearly articulate the structure, theories and concepts to be covered, the outcomes to be achieved, the projected benefits to individuals and organisations, and the resources included (Chimienti et al., 2019).

It emphasises the benefits of cultural diversity and does not identify specific groups for negative stereotypes or use dividing language. Good multicultural competency training encourages participants to be aware of making assumptions about cultural influences or generalisations. It encourages the development of positive attitudes towards cultural and linguistic differences, ideas of justice and inclusion, and the removal of barriers by exploring areas of similarity (Barone et al., 2019). Good training promotes constant sensitive and respectful communication and emphasises personal reflection as a key aspect of developing multicultural competency. Organisations that have honestly evaluated their cultural competence should be able to formulate training goals and objectives. Some organisations may require an introductory program to introduce employees to broad topics and concepts, while others need tangible tools and strategies to develop workforce diversity or improve customer outcomes. Good multicultural competency training programs clearly state how they will meet the needs of the organisation or the people they teach. They clearly outline the results that organisations and individuals can expect (Rodríguez-Peña, 2021). Regardless of the teaching method, high-quality curricula give participants the opportunity to interact with theories and concepts and engage in self-reflection. These also include setting goals for personal or organisational change and suggestions for follow-up or evaluation (Schinkel, 2018).

There are high-quality online training programs that provide opportunities for interaction and selfreflection, although online programs are ideal for classroom training. Online programmes can introduce the concept of cultural and linguistic diversity to a large number of employees and signal that the organisation values diversity. However, as the Australian Commissioner for Human Rights on discrimination has put it, the best approach seems to be one that transforms people's experiences. Eye-to-eye interaction often transforms the experience, as there is an opportunity to engage in dialogue, ask questions, and practice selfreflection. It is presented in a professional, interesting and accessible format. Multicultural competency training can include dense theoretical content and complex concepts. For effective communication and good cultural competencies, the material is presented excitingly and pleasantly for participants of training programmes. It includes pre- and post-training assessments. The basic assessment means that the content of the training can be adapted accordingly and allows providers of higher educational institutions to assess 
existing levels of multicultural competency. Qualitative survey and evaluation processes also provide both qualitative and quantitative data to measure learning effectiveness (Oyelere et al., 2020).

Although there is no established profile for qualified teachers of multicultural competency, they share a number of common skills and properties. Qualified trainers can create an environment where participants feel safe for dialogue and questions, understanding that difficult or uncomfortable problems may arise. They are able to navigate sensitive issues and ensure that the discussion remains respectful. Qualified trainers are also experts in subject areas, although not all trainers acquire knowledge in the same way. Some may have significant education, while others may have experience as a tourism employee. They may have experienced discrimination, racism, or first rejection and incorporated their own experiences into their studies. They may have extensive experience working internationally or interacting with different cultures. Qualified trainers use a wide range of materials and sources and demonstrate a commitment to continuous personal and professional development. Importantly, qualified trainers show genuine interest in other cultures. They are truly committed to cultural and linguistic diversity and inclusion and believe in the benefits of learning cultural competencies.

A number of questions can affect the likelihood that an organisation will start learning about multicultural competency. An organisation may not consider multicultural competency because its management does not recognise its value or is not committed to improving multicultural competency when faced with competing priorities. Organisations that wish to receive multicultural competency training may not be able to find quality resources and information, do not know how to find or identify a suitable multicultural competency coach, or do not know how to verify the quality of a potential service provider (Favell, 2019). Managers with no experience often underestimate the need to participate in multicultural competency training. They usually believe that they already have the necessary skills and behaviours or fail to see how cultural competence training will improve the functioning of the organisation. Even managers who recognise the benefits of cultural and linguistic diversity often don't see how cultural competence is directly linked to their organisation. In fast-paced and demanding jobs, cultural competence is often considered the second most important issue among other competing priorities. Without an organisation's commitment to provide and facilitate staff training opportunities at all levels on multicultural competency, appropriate time or financial resources will not be allocated for training (Li et al., 2019).

While pre- and post-training surveys to gain insight into satisfaction, engagement, and knowledge acquisition are relatively easy to implement and administer, the framework for monitoring and evaluating long-term organisational benefits requires sophisticated strategies. More research, projects and reports require convincing organisations of the tangible benefits and value of learning cultural competencies in the end. Different approaches to teaching cultural competencies in terms of terminology, concepts, content, and presentation make it difficult to establish standards, uniformity, or accreditation. Now, there are no guidelines for training and quality assurance. This meant that there was no oversight or accreditation required to ensure the high quality of evidence-based programs. Similarly, there is no accreditation process for trainers who conduct multicultural competency training. There are no mechanisms to prevent the work of unskilled or low-quality trainers. Poor training can reinforce stereotypes and differences. Organisations and individuals who have a negative experience working with low-quality trainers are less likely to participate in further training. Without a formal accreditation system for cultural competence training programs and trainers, there are limited quality resources and textbooks from major publishers based on diversity and multicultural competency in context (Spencer and Triandafyllidou, 2020).

In the absence of a central place to post information on multicultural competency training, it is difficult for organisations to find high-quality guidelines and resources developed in context. For multicultural competency, there are very few professional development opportunities for trainers who want to go through professional development, or new trainers who are looking for a path to the sector. There are few official ways for new coaches to enter the sector and few opportunities to connect with other professionals. There are also limited opportunities or platforms for professionals to share resources and Information (d'angelo and Priulla, 2020). Diversity is closely linked to cultural competence, especially in the context of a diverse workforce and diversity management. Internally, managers and staff should have the skills to manage and collaborate with different teams, while externally, employees should have the skills to interact with a wide 
range of clients, clients, and stakeholders. In the context of employee management and customer service, diversity is defined as all significant differences between people, including the perception of differences that need to be considered in a particular situation and circumstances. Often, the most significant differences are the least obvious, such as thinking styles or beliefs and values. There are many dimensions of diversity that may be less important for different business functions and relationships - literacy, gender, religion, communication style, caregiver roles, and management style.

In this context, diversity management emphasises recognising differences and their impact on business functions the ongoing process of incorporating recognition of differences between the workforce and customers into all core business management functions, communications, processes, and services to create an honest, harmonious, inclusive, creative, and effective organisation. Inclusion occurs when diversity is respected (i.e., different ages, cultural backgrounds, and genders) and contributes to organisational success. Concerning organisational policies and strategies, the use of "diversity" and "inclusion" often refers to diversity in many forms, including taking into account culture, disability, gender, and sexual orientation (Barone and Assirelli, 2020). The first stage of the project was to review the literature on cultural competence as a theoretical and academic concept. The literature was included in the review and examined cultural competence as a concept or theory, the development of terminology and diversity, and inclusion in the Australian context. Many materials were identified that provided an appropriate discussion of multicultural competency and were listed in the references.

Research of the cultural competence training programme. Following a review of the conceptual and theoretical literature, the project included a study of the cultural competence training programs currently offered. This stage included developing a table that listed and compared courses based on the course name, price, duration, format, learning outcomes, and accreditation, where appropriate. Research shows that culturally and linguistically diverse people experience many barriers, including racism and discrimination, in recruitment and promotion. For example, candidates with names associated with a particular ethnicity must apply for a significantly larger number of vacancies before being shortlisted for an interview. Compared to applicants with Anglo-Celtic names, aboriginal and Torres island residents must submit 35\% more applications, candidates with Chinese names must submit $68 \%$ more, Italian names require $12 \%$ more, and "Middle Easterners" - 7\% more applications.

Migrants of origin are forced to use strategies that reduce the emphasis on racial or cultural background, for example, disclosure of their name and lack of work experience or qualifications - even if this applies to the application (Righard, 2021). These types of barriers are not limited to just the recruitment point. Employees should have equal access to promotion and progress, regardless of their cultural or linguistic background. In many industries, including financial services, hospitality, and the Commonwealth civil service, cultural and linguistic diversity is noticeable in mid-level positions and technical specialties, but is absent in senior management and executive roles. A diverse organisation requires that diversity be visible at all levels of the organisation. Along with equal employment opportunities, a multicultural society also requires organisations to provide products and services that meet the needs of a culturally and linguistically diverse consumer base. Research and consultation on this report and other work on access, equity and inclusion show that the provision of services by government, commercial and community sectors does not produce fair results for Australian consumers who recognise culturally and linguistically diverse (Caputo et al., 2019).

\section{CONCLUSIONS}

Educating the multicultural competency of future tourist support specialists (guides) in higher educational institutions is an important pedagogical problem. At the university organisational level, multicultural competency requires precise action and commitment to implementation and evaluation in order to create a workplace that is culturally and linguistically diverse. For example, higher educational organisations with high levels of multicultural competency have recruitment strategies that identify inherent biases; and have processes that help them understand the cultural profiles of the communities they serve. Policies aimed at developing cultural and linguistic diversity can refer to a set of documents or be combined 
into a plan, for example, a multicultural access and equity plan. Although the format may vary from one organisation to another, a quality policy of cultural and linguistic diversity should include a common goal and objectives, as well as support for all actions, initiatives or results.

It is important to define a convenient and efficient schedule and appoint a responsible department or senior employee of higher educational institution, who will be responsible for everything. Multicultural competency curricula help individuals, organisations develop the skills, and tools needed to nurture, manage, and promote diverse teams, and are a key aspect of diverse and inclusive practices. Multicultural competency training, which is proposed to be included in the higher education professional training programs of future tourist support specialists (guides), varies greatly in content, delivery format, duration and price. Some training programmes are specific to a certain sector or professional role, or focus on specific cultural or linguistic communities. Without an official standard or accreditation, the terminology has evolved unevenly around multicultural competency. Although curricula are promoted under a number of different names, cultural competence is most widely used by Ukrainian and international professionals.

\section{REFERENCES}

Ahmed, T., Chandran, V., Klobas, J.E., Liñan, F., \& Kokkalis, P. (2020). Entrepreneurship education programmes: How learning, inspiration and resources affect intentions for new venture creation in a developing economy. The International Journal of Management Education, 18(1), Article number 100327.

Alam, M., Nasir, N., \& Rehman, C. (2020). Intrapreneurship concepts for engineers: A systematic review of the literature on its theoretical foundations and agenda for future research. Journal of Innovation and Entrepreneurship, 9, Article number 8.

Anderson, B. (2019). New directions in migration studies: Towards methodological de-nationalism. Comparative Migration Studies, 7, Article number 36.

Barone, C., \& Assirelli, G. (2020). Gender segregation in higher education: An empirical test of seven explanations. Higher Education, 79(1), 55-78.

Barone, C., Schizzerotto, A., Assirelli, G., \& Abbiati, G. (2019). Nudging gender desegregation: A field experiment on the causal effect of information barriers on gender inequalities in higher education. European Societies, 21(3), 356-377.

Caputo, F., Cillo, V., Candelo, E., \& Liu, Y. (2019). Innovating through digital revolution: The role of soft skills and big data in increasing firm performance. Management Decision, 57(8), 2032-2051.

Chimienti, M., Bloch, A., Ossipow, L., \& de Wenden, C.W. (2019). Second generation from refugee backgrounds in Europe. Comparative Migration Studies, 7, Article number 40.

Chimienti, M., Guichard, E., Bolzman, C., Le \& Goff, J-M. (2021). How can we categorise "nationality" and "second generation" in surveys without (re)producing stigmatisation. Comparative Migration Studies, 9, Article number 29.

D’Angelo, N., \& Priulla, A. (2020). Estimating the number of changepoints in segmented regression models: Comparative study and application. Retrieved from https://papers.ssrn.com/sol3/papers.cfm?abstract_id=3724965

Dahinden, J., \& Anderson, B. (2021). Exploring new avenues for knowledge production in migration research: A debate between Bridget Anderson and Janine Dahinden pre and after the burst of the pandemic. Swiss Journal of Sociology, 47(1), 27-52.

Dahinden, J., Fischer, C., \& Menet, J. (2020). Knowledge production, reflexivity, and the use of categories in migration studies: Tackling challenges in the field. Ethnic and Racial Studies, 44(4), 535-554.

Favell, A. (2019). Integration: Twelve propositions after Schinkel. Comparative Migration Studies, 7(1), Article number 21.

Kovalenko, O.V. (2020). Culturological approach in modern training of tourist support specialists (guides). In Tourism of the XXI century: Global challenges and civilization values: II 
International scientific and practical conference (pp. 657-662). Kyiv: Kyiv National University of Trade and Economics.

Li, K., Zhang, P., Hu, B.Y., Burchinal, M.R., Fan, X., \& Qin, J. (2019). Testing the "thresholds" of preschool education quality on child outcomes in China. Early Childhood Research Quarterly, 47, 445-456.

Oyelere, S.S., Bouali, N., Kaliisa, R., Yunusa, A.A., \& Jimoh, E.R. (2020). Exploring the trends of educational virtual reality games: A systematic review of empirical studies. Smart Learning Environments, 7, Article number 31.

Poglia Mileti, F. (2019). Labelling migrants in Switzerland: Social, political and symbolic dimensions. In B. Lüthi \& D. Skenderovic (Eds.), Switzerland and migration: Historical and current perspectives on a changing landscape (pp. 61-78). Cham: Springer.

Priulla, A., D'Angelo, N., \& Attanasio, M. (2021). An analysis of Italian university students' performance through segmented regression models: Gender differences in STEM courses. Genus, 77, Article number 11 .

Righard, E. (2021). Researching the dynamics of national social policy in the globalized society. A proposal of a de-nationalised analytical framework. Swiss Journal of Sociology, 47(1), 137-155.

Rodríguez-Peña, A. (2021). Assessing the impact of corporate entrepreneurship in the financial performance of subsidiaries of Colombian business groups: Under environmental dynamism moderation. Journal of Innovation and Entrepreneurship, 10, Article number 16.

Santoso, R.T.P.B., Junaedi, I.W.R., Priyanto, S.H., \& Santoso, D.S.S. (2021). Creating a startup at a University by using Shane's theory and the entrepreneural learning model: A narrative method. Journal of Innovation and Entrepreneurship, 10, Article number 21.

Schinkel, W. (2018). Against "immigrant integration": For an end to neocolonial knowledge production. Comparative Migration Studies, 6(1), Article number 31.

Spencer, S., \& Triandafyllidou, A. (Eds.). (2020). Migrants with irregular status in Europe. Cham: Springer.

Spini, D., Morselli, D., Elcheroth, G., Gauthier, J-A., Le Goff, J-M., Dasoki, N., . . Rossignon, F. (2019). The LIVES-FORS cohort survey: A longitudinal diversified sample of young adults who have grown up in Switzerland. Longitudinal and Life Course Studies, 10(3), 399-410. 